\title{
PRÁTICAS DE ATENÇÃO AO PARTO E NASCIMENTO: UMA REVISÃO INTEGRATIVA
}

\section{CARE PRACTICES TO LABOR AND BIRTH: INTEGRATIVE REVIEW}

\section{PRÁCTICAS DE CUIDADO AL PARTO Y NACIMIENTO: UNA REVISION INTEGRADORA}

Thayná Champe da Silva ${ }^{1}$, Priscila Bisognin ${ }^{2}$, Lisie Alende Prates ${ }^{3}$, Cleunir de Fatima Candido de Bortoli ${ }^{4}$, Gabriela Oliveira $^{1}$, Lúcia Beatriz Ressel ${ }^{5}$.

\section{RESUMO}

Objetivo: identificar na literatura científica as práticas de atenção ao parto e nascimento desenvolvidas pelos profissionais de saúde no Brasil. Método: Trata-se de uma revisão integrativa, realizada na biblioteca eletrônica SCIELO, na base de dados especializada em enfermagem e na Literatura Latino-Americana e do Caribe em Ciências da Saúde. Resultados: das 172 publicações encontradas, 15 foram incluídas no estudo. Algumas publicações destacam as boas práticas ao parto e nascimento úteis, como apoio, acolhimento e medidas não farmacológicas para alívio da dor, e outras publicações apontaram alguns entraves para efetivar as boas práticas como a episiotomia de rotina. Conclusão: espera-se que esta revisão contribua para a construção de novas pesquisas e na área da saúde, permitindo, dessa forma, reflexão, e fortaleça o papel dos profissionais da saúde, especialmente do enfermeiro.

Descritores: Saúde da mulher; Parto normal; Parto humanizado; Enfermagem.

\section{ABSTRACT}

Objective: To identify the scientific literature about the practices of attention to labor and birth, developed by health professionals in Brazil. Method: It is an integrative review, held in Scielo electronic library, in specialized database in nursing and in the Latin American and Caribbean Literature in Health Sciences. Results: Of the 172 publications found, 15 were included in the study. Some publications highlight good practice in labor and delivery useful as support, hosting and no pharmacological measures for pain relief, and other publications pointed out some obstacles to effect good practices such as routine episiotomy. Conclusion: It is expected that this review will contribute to the construction of new research and in health and thus enable reflection and strengthen the role of health professionals, especially nurses.

Descriptors: Women's health; Natural childbirth; Humanizing delivery; Nursing.

\section{RESÚMEN}

Objetivo: Identificar en la literatura acerca de las prácticas de atención del parto y nacimiento, desarrollado por profesionales de la salud en Brasil. Método: Se trata de un examen integrador, celebrada en la biblioteca electrónica Scielo, en la base de datos especializada en la enfermería y en la Literatura Latinoamericana y del Caribe en Ciencias de la Salud. Resultados: De las 172 publicaciones encontradas, 15 fueron incluidos en el estudio. Algunas publicaciones destacan las buenas prácticas en el trabajo de parto útil como apoyo, alojamiento y medidas no farmacológicas para aliviar el dolor, y otras publicaciones señalaron algunos obstáculos para llevar a cabo buenas prácticas como la episiotomía de rutina. Conclusión: Se espera que este estudio contribua a la construcción de nuevas investigaciones y en la salud y permitir así la reflexión y reforzar el papel de los profesionales de la salud, sobre todo enfermeros.

Descriptores: Salud de la mujer; Parto normal; Parto humanizado; Enfermería.

${ }^{1}$ Acadêmica de Enfermagem, Universidade Federal de Santa Maria/UFSM. ${ }^{2}$ Graduada em Enfermagem. Mestranda em Enfermagem pela Universidade Federal de Santa Maria - UFSM. ${ }^{3}$ Graduada em Enfermagem. Doutoranda pelo Programa de Pós-Graduação em Enfermagem pela Universidade Federal de Santa Maria - UFSM. ${ }^{4}$ Graduada em Enfermagem. Mestranda em Enfermagem pela Universidade Federal de Santa Maria/PPGEnf/UFSM. ${ }^{5}$ Graduada em Enfermagem. Doutora em Enfermagem. Docente Associada pela Universidade Federal de Santa Maria/UFSM.

Como citar este artigo:

Silva TC, Bisognin P, Prates LA, et al. Práticas de atenção ao parto e nascimento: uma revisão integrativa Labor And Birth Care. Revista de Enfermagem do Centro-Oeste Mineiro. 2017;7:e1294. [Access___]; Available in:___.Doi: http://dx.doi.org/10.19175/recom.v7i0.1294 


\section{INTRODUÇÃO}

O parto é considerado uma experiência repleta de significados construídos a partir da singularidade e cultura de cada parturiente ${ }^{(1)}$. Por isso, a assistência obstétrica humanizada visa à promoção do respeito aos direitos da mulher e da criança, com condutas baseadas em evidências científicas. As ações voltadas à humanização do parto e nascimento proporcionam reflexões sobre a assistência obstétrica que foram adotadas no passado, quando um menor número de intervenções era realizado ${ }^{(2)}$. Portanto, o cuidado realizado nesse acontecimento pode implicar de modo positivo ou negativo no processo de gestação e parto da mulher, do recém-nascido, do companheiro ou família.

Em 1996, a Organização Mundial da Saúde (OMS) desenvolveu uma classificação das práticas comuns na condução do parto normal, orientando para o que deve e o que não deve ser realizado no processo do parto e nascimento. Essa classificação foi baseada em evidências científicas concluídas através de pesquisas realizadas no mundo todo, com o intuito de estabelecer práticas adequadas e seguras para a assistência obstétrica, garantindo uma atenção materno-infantil qualificada, humanizada e segura. Nessa direção, as recomendações foram classificadas em quatro categorias: práticas demonstradamente úteis e que devem ser estimuladas; práticas claramente prejudiciais ou ineficazes e que devem ser eliminadas; práticas sem evidências suficientes para apoiar uma recomendação clara e que devem ser utilizadas com cautela até que mais pesquisas esclareçam a questão; práticas frequentemente usadas de modo inadequado ${ }^{(3)}$.

Dito isso, o Ministério da Saúde (MS), com o objetivo de dar continuidade na classificação desenvolvida em 1996 pela OMS, implantou em 2000 um amplo processo de humanização da assistência obstétrica por meio do Programa de Humanização do Pré-Natal e Nascimento (PHPN). Essa estratégia tem como desígnio o resgate da atenção obstétrica qualificada, integrada e humanizada no pré-natal, parto e puerpério, com o envolvimento dos estados, municípios e instituições de saúde ${ }^{(4)}$. Em 2001, o MS publicou o manual "Parto, Aborto e Puerpério: assistência humanizada à mulher", fundamentado na ciência e nas recomendações da OMS, no qual reconhece a importância da humanização da assistência à mulher durante o ciclo gravídico-puerperal para melhorar a qualidade da atenção prestada, inclusive estimulando a presença de um acompanhamento ou suporte psicossocial durante o trabalho de parto ${ }^{(5)}$.

A busca pelo cuidado humanizado no parto e nascimento é uma temática de grande interesse, ainda que os caminhos percorridos para se alcançar tal objetivo seja um grande entrave. Portanto, com base na proposta de humanização, a ampliação dessas práticas na assistência à parturição prevê atitudes e condutas dos profissionais da saúde que contribuam para reforçar o caráter de atenção à saúde como um direito de todas as mulheres. Contudo, a realidade de muitas instituições de saúde demonstra resistências a essas recomendações, principalmente nos Centros Obstétricos ${ }^{(6)}$.

Atualmente, o modelo de cuidado utilizado pela enfermagem obstétrica e neonatal encontrase pautado na humanização da assistência realizada à mulher e ao recém-nascido, tendo como embasamento as políticas públicas de saúde, fazendo uso de tecnologias apropriadas, necessárias e fundamentadas, valorizando a cultura, crenças e modos de vida de cada mulher $^{(7)}$. Baseado nisso, a enfermagem desempenha papel de grande importância na construção de um panorama mais promissor para atenção ao parto e nascimento do Brasil.

Diante do exposto, este estudo de revisão justificou-se pela possibilidade de síntese e análise da literatura científica sobre as práticas de atenção ao parto e nascimento desenvolvidas pelos profissionais de saúde no Brasil cujo objetivo foi de 'identificar na literatura científica as práticas de atenção ao parto e nascimento desenvolvidas por profissionais de saúde no Brasil'.

\section{MÉTODO}

Trata-se de uma revisão integrativa, método de pesquisa que tem como finalidade reunir e sintetizar resultados de pesquisas sobre um delimitado tema, de maneira ordenada e sistemática. Refere-se a um método que permite o aprofundamento do conhecimento a respeito do tema investigado, a síntese de múltiplos estudos publicados e conclusões gerais a respeito de uma área particular de estudo. É importante destacar que através dessa estratégia metodológica os profissionais de diversas áreas de atuação na saúde têm rápido acesso aos resultados mais relevantes de pesquisas que podem fundamentar as condutas ou a tomada de decisão, proporcionando um saber crítico e um fazer qualificado ${ }^{(8)}$. 
Para a realização da revisão, percorreramse as etapas: estabelecimento do objetivo da revisão integrativa; estabelecimento dos critérios para a seleção dos estudos; definição das informações a serem extraídas dos artigos selecionados; análise dos resultados; apresentação e discussão dos resultados ${ }^{(8)}$. A questão que orientou a pesquisa foi: Quais são as boas práticas de atenção ao parto e nascimento disponíveis na literatura científica desenvolvidas pelos profissionais de saúde no Brasil?

Os critérios de inclusão foram: pesquisas originais realizadas no Brasil, publicadas nos idiomas inglês, português ou espanhol; disponíveis online e gratuitas; estudos a partir de 1996. Destaca-se o marco temporal adotado, haja vista uma publicação lançada pela OMS em 1996 a respeito das boas práticas de atenção ao parto e nascimento. Como critérios de exclusão: trabalhos que não contemplavam a temática ou não respondiam à questão orientadora.

A busca bibliográfica ocorreu no mês de setembro de 2015, na base de dados Bibliográficos Especializada na Área de Enfermagem do Brasil (BDENF), na Scientific Electronic Library Online (SciELO) e na Literatura Latino-Americana em Ciências de Saúde (LILACS).

A seguir a figura mostra o processo de busca dos estudos.

Figura 1 - Fluxograma de busca e inclusão dos estudos.

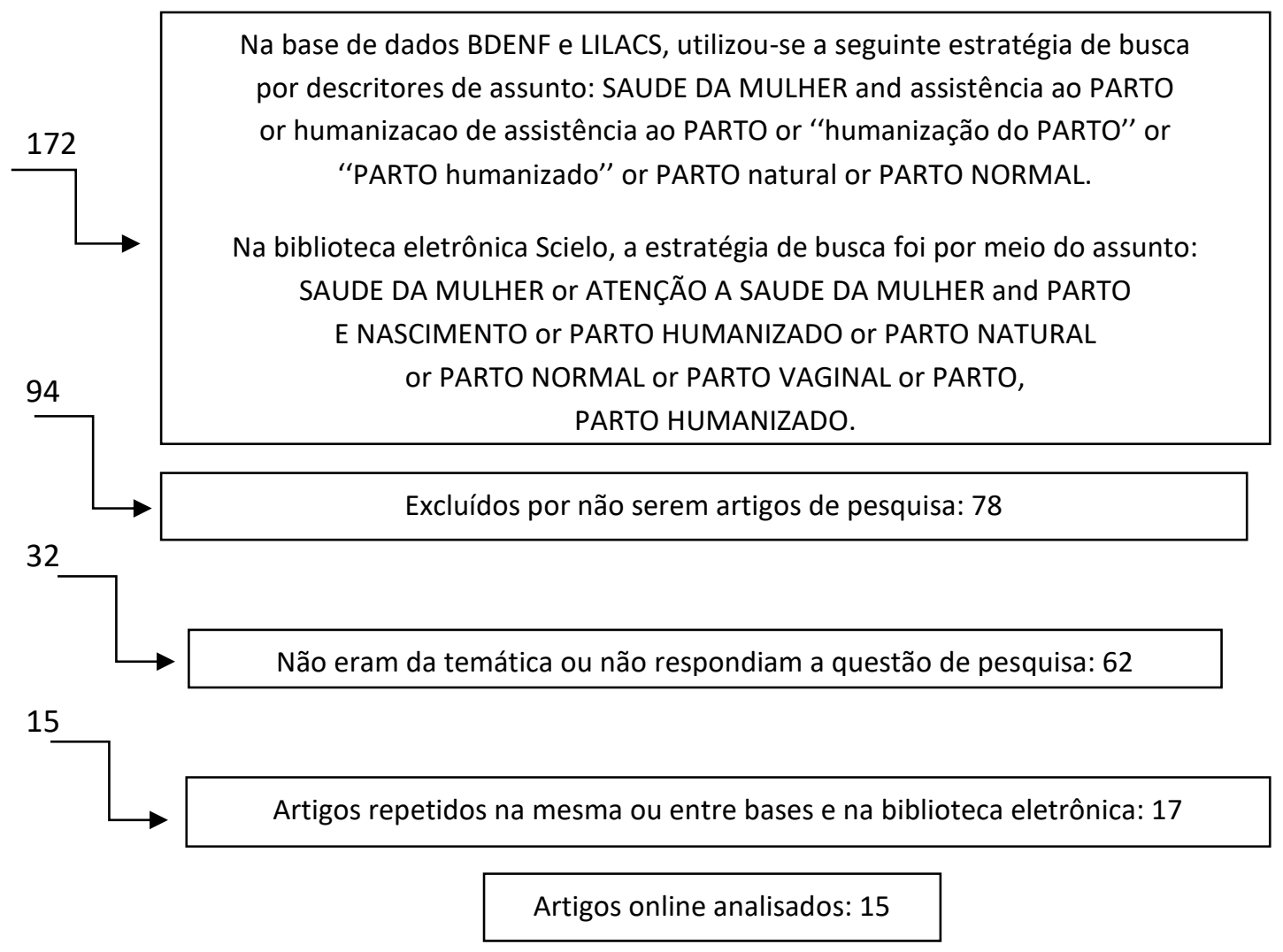

Os artigos selecionados ainda foram lidos na íntegra e dispostos em um quadro analítico, contendo a identificação do artigo, objetivo, método empregado e as práticas de atenção ao parto e nascimento realizadas pelos profissionais de saúde. Este quadro foi desenvolvido para organização dos dados e posterior análise. A análise textual qualitativa foi empregada para análise e avaliação dos estudos, de modo que o conteúdo da análise se formou a partir das produções científicas ${ }^{(9)}$. Esse método envolve a análise de textos e discursos, considerando que este é uma formação discursiva ${ }^{(9)}$. Esse tipo de análise mostra-se útil, quando se pretende fazer uma síntese em que as principais questões sejam reconstruídas e sistematizadas. Ainda se ressalta que os princípios éticos do estudo foram preservados, tendo sido referenciados adequadamente todos os autores das pesquisas consultadas, conforme a Lei dos Direitos Autorais ${ }^{(10)}$. 


\section{RESULTADOS E DISCUSSÃO}

Dentre os 15 artigos analisados, 14 (93,3\%) consistiam em pesquisas com abordagem qualitativa, uma pesquisa $(6,6 \%)$ como quantitativa. A maioria das publicações foi realizada na região Sudeste com seis (40\%) estudos e Nordeste com cinco $(33,3 \%)$ estudos, seguida da região Sul com quatro $(26,6 \%)$.

Quanto aos anos de publicação, identificaram-se quatro publicações no ano de $2012(26,6 \%)$, três (20\%) publicação no ano de 2011, duas (13,3\%) no ano 2009 e 2013, uma (6,6\%) nos anos de 2004, 2005, 2010 e 2015. Salienta-se que nos últimos anos as questões relacionadas à humanização no parto e resgate das boas práticas de atenção ao parto e nascimento se intensificaram, possivelmente justificando o número de publicações em anos recentes. Pode-se citar o manual de Boas Práticas de Atenção ao Parto e Nascimento ${ }^{(3)}$ e o Programa de Humanização no Pré-Natal e Nascimento, no âmbito do Sistema Único de Saúde.

Entre os periódicos científicos que se destacaram na publicação dos artigos, tem-se a Revista de Pesquisa: Texto e Contexto com três publicações (20\%). Escola Anna Nery Revista de
Enfermagem, Revista de Enfermagem UERJ, Revista de Enfermagem de Brasília e Revista da Rede de Enfermagem do Nordeste com duas publicações cada (13,3\%). A revista Cuidado é Fundamental Online, Revista de Enfermagem da UFSM, Cogitare Enfermagem e Revista de Enfermagem da USP com uma publicação cada $(6,6 \%)$.

Quanto aos cenários, verificou-se que os estudos foram realizados em centros obstétricos e em maternidades de hospitais privados e públicos. Destaca-se a Enfermagem como a área profissional que mais produziu estudos, com 14 $(93,3 \%)$ sobre a temática, seguida pela Medicina 1 estudo $(6,6 \%)$ estudos.

Considera-se compreensível esse número de estudos realizados pela enfermagem, tendo em vista a atuação desses profissionais nesse cenário. Acredita-se que a enfermagem tenha um papel essencial na mudança das práticas de atenção ao parto, pois o enfermeiro que tem uma formação humanista e solidária poderá servir de articulador na operacionalização da Política de Humanização do Parto e Nascimento ${ }^{(24)}$.

A seguir, apresenta-se a Figura 2 com a síntese dos resultados encontrados nos estudos.

Figura 2 - Síntese dos estudos incluídos na revisão integrativa segundo as práticas de cuidado realizadas pelos profissionais de saúde.

\begin{tabular}{|c|c|}
\hline A12 & Práticas de atenção ao parto e nascimento desenvolvidas por profissionais de saúde no Brasil. \\
\hline A13 & $\begin{array}{l}\text { É necessário o acolhimento inicial das parturientes e seus acompanhantes nas unidades obstétricas como } \\
\text { uma medida de alívio do desconforto oriundo do processo parturitivo; informações. }\end{array}$ \\
\hline A14 & Relações de respeito e carinho, informações, infraestrutura e recursos. \\
\hline A15 & $\begin{array}{l}\text { Acolhimento parturiente e acompanhante; escolha do local do parto; informações; plano individual de } \\
\text { cuidado. }\end{array}$ \\
\hline A16 & Respeito à escolha do acompanhante; apoio, utilização de método não farmacológico de alívio da dor. \\
\hline A17 & $\begin{array}{l}\text { Acompanhante, monitoramento do bem-estar físico da mulher, informações, privacidade, plano individual, } \\
\text { habilidades técnicas evitando uso de técnicas rotineiras desnecessárias, embasamento científico, } \\
\text { valorização da mulher. }\end{array}$ \\
\hline A18 & Monitoramento do parto, respeito à autonomia, informações, métodos não invasivos, liberdade e respeito. \\
\hline A19 & $\begin{array}{l}\text { Acolhimento e a formação de vínculo com a gestante e familiar; respeitosa, individualizada e priorizando o } \\
\text { aspecto psicológico e cultural; evitar práticas rotineiras. }\end{array}$ \\
\hline A20 & Episiotomia, tricotomia, ocitocina, posição litotônica. \\
\hline A21 & Apoio, informações, monitoramento do bem-estar físico da mulher, oferta de líquidos, acompanhante. \\
\hline A22 & Relaxamento, autonomia, métodos não farmacológicos, monitorização. \\
\hline A23 & $\begin{array}{l}\text { Respeito à autonomia e à fisiologia feminina, com base em evidências científicas, acompanhamento e } \\
\text { monitorização, SUS. }\end{array}$ \\
\hline A24 & $\begin{array}{l}\text { Privacidade e intimidade; Acompanhamento; Relação da equipe/parturiente e familiar; Orientações sobre } \\
\text { o parto; Escolha do tipo de parto; Medidas de higiene; Contato mãe/filho; Estímulo à amamentação; } \\
\text { O bebê mama na primeira hora de vida; Episiotomia. }\end{array}$ \\
\hline A25 & $\begin{array}{l}\text { Enteroclisma, episiotomia, falta de autonomia por parte da parturiente, informações falhas, são utilizadas } \\
\text { técnicas de relaxamento no pré-parto. }\end{array}$ \\
\hline A26 & Relaxamento, contato pele a pele mãe-filho, técnicas não farmacológicas, autonomia, informações claras, SUS. \\
\hline
\end{tabular}


Ao analisar os artigos incluídos nesta revisão, foi possível identificar duas direções opostas de cuidado à parturiente, que estão relacionadas, principalmente, às práticas humanizadas na atenção ao parto e nascimento e em contraponto às práticas em que os profissionais de saúde utilizam práticas prejudiciais, no contexto do Parto e Nascimento, obtendo-se assim obstáculos para a realização de boas práticas de atenção ao parto e nascimento no cenário brasileiro. Aproximadamente, 11 estudos $^{(12-18,20-21,23,26)}, \quad(73,3 \%) \quad$ analisados mencionam evitar o uso de práticas rotineiras e desnecessárias.

A partir da revisão, compreende-se que o processo de humanização do parto e nascimento tem início durante as primeiras consultas de prénatal através de informações, esclarecimentos e acolhimento realizados pelos profissionais de saúde, além de orientações acerca do processo de parturição, cuidados com recém-nascido e seus direitos perante a lei ${ }^{(11)}$.

Algumas publicações destacam as boas práticas ao parto e nascimento demonstradamente úteis e que devem ser estimuladas: valorização da singularidade de cada parturiente e família ${ }^{(13-18,20-21,23,26)}$; respeito aos direitos de escolha da parturiente ofertando liberdade e privacidade ${ }^{(12,14,16-17,23-24)}$. $O$ atendimento baseado na integralidade e equidade da assistência ${ }^{(23,26)}$, conforme as diretrizes do SUS e as políticas públicas de saúde direcionadas às mulheres. Essas declarações demonstram a necessidade da presença de um profissional de saúde habilitado para a construção e estabelecimento do sentimento de segurança e apoio à mulher, mesmo com a presença do acompanhante. Para isso, faz-se necessário investimentos na formação dos profissionais que atuam no processo de parto nascimento, com enfoque além das tecnologias adequadas e úteis ao atendimento ao parto, mas também embasado nos pressupostos da humanização no processo de parturição.

$\mathrm{O}$ apoio dos profissionais de saúde durante o processo de parturição configura-se como importante e reconfortante para as mulheres. Manifestar-se preocupado com o bem-estar e disposto a cuidar e escutar é imprescindível para a criação de vínculo e afeição, assim o processo de parto torna-se menos apavorante, transformando-o em um momento de amor, cuidado e conforto, sempre valorizando a singularidade de cada mulher e família. Muitas vezes não são necessárias palavras ou ações, basta um olhar que transpasse confiança ${ }^{(27)}$.

$\mathrm{O}$ acolhimento pelos profissionais de saúde é fundamental para a criação de vínculo com a mulher e sua família, a partir de ações e condutas que expressem interesse, disponibilidade e respeito, contribuindo, assim, na redução de estresse, medos e angústia diante da proximidade do trabalho de parto ${ }^{(13)}$. Os profissionais de saúde também destacam que a presença do acompanhante contribui como apoio emocional para a parturiente. Essa percepção da participação do acompanhante se ancora no apoio emocional e tem sua maior expressão na transmissão de maior segurança e conforto para a parturiente, em um momento em que a solidão e o medo podem se fazer presentes ${ }^{(12,14-15,20,22)}$. Nessa perspectiva, revela-se que o suporte do acompanhante no processo da parturição poderá proporcionar à mulher sentimentos positivos, como a sensação de amparo, a coragem, a tranquilidade e o conforto, com consequente redução do medo e da ansiedade ${ }^{(15)}$.

Acredita-se que a presença e o apoio do acompanhante durante o trabalho de parto e parto contribuem significativamente para que esse evento decorra da melhor forma possível, com tranquilidade e segurança. Com base nos achados da evidência científica, a Organização Mundial de Saúde (OMS) publicou, em 1996, um guia prático para assistência ao parto normal, no qual classificou o apoio empático fornecido pelos prestadores de serviço e o respeito à escolha da mulher sobre seus acompanhantes no parto como uma prática útil e que deve ser estimulada.

A participação do acompanhante na humanização do parto e nascimento, mesmo sendo amparada legalmente, ainda é um processo em construção e envolve diversos aspectos, entre eles as condições físicas ambientais dos hospitais, a qualificação dos profissionais de saúde para o acolhimento do acompanhante e atitudes de submissão das gestantes diante de seus direitos ${ }^{(15)}$. O suporte do acompanhante no processo da parturição poderá proporcionar à mulher sentimentos positivos, como a sensação de amparo, coragem, tranquilidade e conforto, com consequente redução do medo e da ansiedade. 0 estabelecimento de uma relação de confiança com os profissionais de saúde fortalece sentimentos positivos que tranquilizam a parturiente ${ }^{(12)}$. Reforçando 0 exposto, o 
Ministério da Saúde em 2000, através da portaria no 569, tornou público a regulamentação em que as instituições de saúde devem possibilitar à gestante $\mathrm{o}$ direito a ter um acompanhante durante o processo de pré-parto e parto ${ }^{(28)}$.

Dentre as publicações analisadas, os profissionais de saúde apontaram alguns entraves para efetivar as boas práticas de atenção ao parto e nascimento em seus serviços, entre elas destacam-se: o cuidado centrado no modelo biomédico, desvalorizando a autonomia da mulher e repercutindo no protagonismo da mulher; falta de leitos e estrutura organizacional do ambiente físico das instituições deficiente; número insuficiente de funcionários capacitados para atuar na assistência à mulher no processo de parturição, além do despreparo do acompanhante e da família para lidar com os sentimentos permeados nesse contexto de parto e nascimento ${ }^{(19,22,24-25)}$. Somando-se a isso, evidencia-se um núcleo de ações em que os profissionais de saúde utilizam práticas prejudiciais ou ineficazes, sem embasamento científico suficiente, desrespeitando os direitos e preferências dessas mulheres. Dentre as quais, podemos citar o uso de enteroclisma ${ }^{(25)}$, uso da tricotomia ${ }^{(19,22,25)}$, infusão de soro com ocitocina, posição do parto e uso rotineiro da episiotomia ${ }^{(19,}$ 22,24-25). A OMS e o MS, baseados nas evidências científicas, recomendam o uso restrito da episiotomia e classificam seu uso rotineiro e liberal como uma prática claramente prejudicial, que deve ser desestimulada, sendo indicada somente em cerca de $10 \%$ a $15 \%$ dos casos $^{(25)}$.

É importante ressaltar que a Enfermagem tem participado desde as primeiras discussões acerca da saúde da mulher, sendo a partir de movimentos sociais ou no processo de implantação e consolidação das políticas públicas vigentes no país em defesa da humanização no pré-natal, parto e nascimento ${ }^{(13,17,21,26)}$. Diante disso, o MS tem criado portarias que favorecem a atuação do profissional enfermeiro na atenção integral à saúde da mulher, privilegiando o período gravídico puerperal por entender que essas medidas são fundamentais para a diminuição de intervenções desnecessárias e que geram risco, tanto em centros obstétricos como em casas de parto ou maternidades, favorecendo o cuidado integral à mulher e recém-nascido ${ }^{(29)}$.

Portanto, faz-se necessária a sensibilização e conscientização dos profissionais de saúde, com intuito de reflexão acerca do modelo de cuidado à saúde da mulher durante o processo de parturição.

\section{CONCLUSÃO}

Passadas quase duas décadas da implantação do manual da atenção ao parto e nascimento, observa-se que ainda existem instituições de saúde em que suas práticas de atenção ao parto e nascimento estão centradas no atendimento pautado em ações intervencionistas, moduladas a partir do modelo biomédico. Esta revisão suscitou a urgente necessidade de se refletir e garantir uma atenção materno-infantil qualificada, em que as práticas prejudiciais ou ineficazes sejam suprimidas e que práticas sem evidências suficientes para apoiar uma recomendação clara devem ser utilizadas com cautela até que mais pesquisas esclareçam a questão.

Salienta-se que, além da importância das políticas públicas vigentes, é necessário implementar capacitações e atualização profissional, somando-se a isso a mudança na formação dos profissionais de saúde que atuam no processo de parto e nascimento.

Espera-se que esta revisão contribua para a construção de novas pesquisas nessa área no Brasil, com enfoque multiprofissional, e que assim esse contexto seja fortalecido. Observouse, também, o papel do profissional enfermeiro nesse cenário, como articulador nesse contexto, somando-se seu conhecimento teórico e manejo nas situações emocionais vivenciadas pela mulher, acompanhante e família.

\section{REFERÊNCIAS}

1. Lopes CV, Meincke SMK, Carraro TE, Soares MC, Reis SP, Heck RM. Experiências vivenciadas pela mulher no momento do parto e nascimento de seu filho.Cogitare enferm. 2009;14(3):484-90. Disponível

em: http://revistas.ufpr.br/cogitare/article/view/1617 8/10697

2. Gallo RBS, Santana LS, Marcolin AC, Ferreira $\mathrm{CHJ}$, Duarte G, Quintana SM. Recursos nãofarmacológicos no trabalho de parto: protocolo assistencial. Femina. 2011 jan; 39(1).

3. Organização Mundial da Saúde. Maternidade segura. Assistência ao parto normal: um guia prático. Genebra: Organização Mundial da Saúde; 1996.

4. Ministério da Saúde (BR). Secretaria de Políticas de Saúde. Programa humanização no 
pré-natal e nascimento. Brasília: Ministério da Saúde; 2000a.

5. Ministério da Saúde (BR). Parto, aborto e puerpério: assistência humanizada à mulher. Brasília: Ministério da Saúde; 2001.

6. Nagahama EEI, Santiago SM. Práticas de atenção ao parto e os desafios para humanização do cuidado em dois hospitais vinculados ao Sistema Único de Saúde em município da Região Sul do Brasil. Cad. saúde pública. 2008;24(8):1859-68. Disponível em: http://www.scielo.br/pdf/csp/v24n8/14.pdf

7. Silva LR, Christoffel MM, Souza KV. História, conquistas e perspectivas no cuidado à mulher $\mathrm{e}$ à criança. Texto \& contexto enferm. 2005;14(4):585-93. Disponível em: http://www.scielo.br/pdf/tce/v14n4/a16v14n4.pdf 8. Mendes KDS, Silveira RCCP, Galvão CM. Revisão integrativa: método de pesquisa para a incorporação de evidências na saúde e na enfermagem. Texto \& contexto enferm. 2008;17(4):758-64. Disponível em: http://www.scielo.br/pdf/tce/v17n4/18.pdf

9. Moraes R. Uma tempestade de luz: a compreensão possibilitada pela análise textual discursiva. Ciênc. educ. (Bauru). 2003;9(2):191$210 . \quad$ Disponível em: http://www.scielo.br/pdf/ciedu/v9n2/04.pdf

10. Ministério da Saúde (BR). Lei no 9.610, de 19 de fevereiro de 1998. Altera, atualiza e consolida a legislação sobre direitos autorais e dá outras providências. Diário Oficial da República Federativa do Brasil. Brasília: Ministério da Saúde; 1998.

11. Wolff LR, Waldow VR. Violência consentida: mulheres em trabalho de parto e parto.Saúde soc. 2008;17(3):138-51. Disponível em: http://www.revistas.usp.br/sausoc/article/view/7 604/9128

12. Santos LM, Pereira SSC, Santos VEP, Santana RCB, Melo MCP. Relacionamento entre profissionais de saúde e parturientes: um estudo com desenhos. Rev. enferm. UFSM. 2011; 1(2):225-37. Disponível em: http://periodicos.ufsm.br/reufsm/article/view/25 $\underline{\text { 88/1635 }}$

13. Cassiano AN, Araujo MG, Holanda CSM, Costa RKS. Percepção de enfermeiros sobre a humanização na assistência de enfermagem no puerpério imediato. Rev. pesqui. cuid. fundam. (Online).2015;7(1):2051-60. Disponível em: http://www.seer.unirio.br/index.php/cuidadofun damental/article/download/3674/pdf 1453.
14. Santos FRP, Tyrrell MAR. A assistência à mulher no pré-parto e parto na perspectiva da maternidade segura. Esc. Anna Nery Rev. Enferm. 2005 abr; 9(1):46-53. Disponível em: http://www.redalyc.org/articulo.oa?id=12772049 $\underline{4006}$

15. Santos LM, Carneiro CS, Carvalho ESS, Paiva MS. Percepção da equipe de saúde sobre a presença do acompanhante no processo parturitivo. Ver. RENE. 2012; 13(5):994-1003. Disponível

em:

http://www.revistarene.ufc.br/revista/index.php/ revista/article/viewFile/1157/pdf

16. Barros LM, Silva RM. Atuação da enfermeira na assistência a mulher no processo de parturição. Texto \& contexto enferm. 2004; 13(3):376-82. Disponível em: http://www.scielo.br/scielo.php?script=sci arttex t\&pid=S0104-07072004000300006

17. Progianti JM, Mouta RJO. A enfermeira obstétrica: agente estratégico na implantação de práticas do modelo humanizado em maternidades. Rev. Enferm. UERJ. 2009; 17(2):165-9. Disponível em: http://www.facenf.uerj.br/v17n2/v17n2a04.pdf

18. Moreira KAP, Araújo MAM, Fernandes AFC, Braga VAB, Marques JF, Queiroz MVO. O significado do cuidado ao parto na voz de quem cuida: uma perspectiva à luz da humanização. Cogitare enferm. 2009; 14(4):720-8. Disponível em:

http://revistas.ufpr.br/cogitare/article/viewFile/1 6389/10869

19. Carvalho VF, Kerber NPC, Busanello J, Costa MMG, Gonçalves BG, Quadros VF. Práticas prejudiciais ao parto: relato dos trabalhadores de saúde do Sul do Brasil. Rev. RENE. 2010; 11(esp):92-8. Disponível em: http://www.revistarene.ufc.br/edicaoespecial/a1 Ov11esp n4.pdf

20. Prata JA, Progianti JM. A influência da prática das enfermeiras obstétricas na construção de uma nova demanda social. Rev. Enferm. UERJ. 2013; 21(1):23-8. Disponível em: http://www.epublicacoes.uerj.br/index.php/enfermagemueri/a rticle/view/6341/4516

21. Progianti JM, Porfírio AB. Participação das enfermeiras no processo de implantação de práticas obstétricas humanizadas na maternidade Alexander Fleming (1998-2004). Esc. Anna Nery Rev. Enferm. 2012; 16(3): 443-50. Disponível em: http://www.scielo.br/scielo.php?script=sci arttex t\&pid=S1414-81452012000300003 
22. Busanello J, Kerber NPC, Sassi-Mendoza RA, Mano PS, Susin LRO, Gonçalves BG. Atenção humanizada ao parto de adolescentes: análise das práticas desenvolvidas em um centro obstétrico. Rev. Bras. enferm. 2011; 64(5):824-32. Disponível em: http://www.scielo.br/scielo.php?script=sci arttex t\&pid=S0034-71672011000500004

23. Malheiros PA, Alves VH, Rangel TSA, Vargens OMC. Parto e nascimento: saberes e práticas humanizadas. Texto \& contexto enferm. 2012; 21(2):329-37. Disponível em: http://www.scielo.br/scielo.php?script=sci arttex t\&pid=S0104-07072012000200010

24. Silva RC, Soares MC, Jardim VMR, Kerber NPC, Meincke SMK. O discurso e prática do parto humanizado de adolescentes. Texto \& contexto enferm. 2013; 22(3):629-36. Disponível em: http://www.scielo.br/scielo.php?script=sci arttex t\&pid=S0104-07072013000300008

25. Carvalho VF, Kerber NPC, Busanello J, Gonçalves BG, Rodrigues EF, Azambuja EP. Como os trabalhadores de um centro obstétrico justificam a utilização de práticas prejudiciais ao parto normas. Rev. Esc. Enferm. USP. 2012; 46(1):30-7. Disponível em: http://www.scielo.br/pdf/reeusp/v46n1/v46n1a0 4.pdf

26. Progianti JM, Costa RF. Práticas educativas desenvolvidas por enfermeiras: repercussões sobre vivências de mulheres na gestação e no parto. Rev. bras. enferm. 2012; 65(2):257-63. Disponível

em:

http://www.scielo.br/scielo.php?script=sci arttex $\underline{\text { t\&pid}=\text { S0034-71672012000200009 }}$

27. Frello AT, Carraro TE. Conforto no processo de parto sob a perspectiva das puérperas. Rev. Enferm. UERJ. 2010;18(3):441-5. Disponível em: http://www.facenf.uerj.br/v18n3/v18n3a18.pdf

28. Ministério da Saúde (BR). Secretaria de Assistência à Saúde. Portaria no 569/GM, de 1ㅇ de junho de 2000: Institui o Programa de Humanização no Pré-Natal e Nascimento, no âmbito do Sistema Único de Saúde. Brasília: Ministério da Saúde; 2000b.

29. Ministério da Saúde (BR). Secretaria de Políticas Públicas de Saúde. Área Técnica de Saúde da Mulher. Parto, aborto e puerpério: assistência humanizada à mulher. Brasília: Ministério da Saúde; 2003.
Nota: Recorte do trabalho de Conclusão de Curso intitulado 'As boas práticas de atenção ao parto e nascimento sob a ótica de enfermeiros'.

Recebido: 03/02/2016

Versão final: $27 / 03 / 2017$

Aprovado em: 28/03/2017

\section{Endereço para Correspondência}

Thayná Champe da Silva

Universidade Federal de Santa Maria - UFSM

Centro de Ciências da Saúde - CCS

Programa de Pós-Graduação em Enfermagem - PPGEnf

Faixa de Camobi, Km 09 - Sala 1302 - Prédio 26

Santa Maria, RS - CEP: 97105-900

E-mail: thaynachampe@hotmail.com 